\title{
State-by-State Investigation of Destructive Interference in Resonance Raman Spectra of Neutral Tyrosine and the Tyrosinate Anion with the Simplified Sum-over-States Approach
}

\section{Citation}

Cabalo, Jerry B., Semion K. Saikin, Erik D. Emmons, Dmitrij Rappoport, and Alán Aspuru-Guzik. 2014. "State-by-State Investigation of Destructive Interference in Resonance Raman Spectra of Neutral Tyrosine and the Tyrosinate Anion with the Simplified Sum-over-States Approach." The Journal of Physical Chemistry A 118 (41) (October 16): 9675-9686. doi:10.1021/jp506948h.

\section{Published Version}

doi:10.1021/jp506948h

\section{Permanent link}

http://nrs.harvard.edu/urn-3:HUL.InstRepos:23670484

\section{Terms of Use}

This article was downloaded from Harvard University's DASH repository, and is made available under the terms and conditions applicable to Open Access Policy Articles, as set forth at http:// nrs.harvard.edu/urn-3:HUL.InstRepos:dash.current.terms-of-use\#OAP

\section{Share Your Story}

The Harvard community has made this article openly available.

Please share how this access benefits you. Submit a story. 


\title{
A State-by-state Investigation of Destructive
}

\section{Interference in Resonance Raman Spectra of Neutral}

\section{Tyrosine and Tyrosinate Anion with the Simplified}

\section{Sum-over-States Approach}

Jerry B. Cabalo ${ }^{* a}$, Semion K. Saikin ${ }^{b}$, Erik D. Emmons ${ }^{c}$, Dmitrij Rappoport $^{b}$, Alán Aspuru-

\author{
$G u z i k^{b}$
}

*Corresponding Author

AUTHOR ADDRESSES: a. U. S. Army ECBC, RDCB-DRI-I/BLDG E5951, 5183 Blackhawk

Road, APG, MD 21010, Phone: 410-436-1760, FAX: 410-436-2742

b. Department of Chemistry and Chemical Biology, Harvard University, 12 Oxford Street, Cambridge, MA 02138.

c. Leidos, Inc., P.O. Box 68, Gunpowder Branch, Aberdeen Proving Ground, Maryland, 210105424, United States..

KEYWORDS: resonance Raman, tyrosine, sum over states, Raman excitation profile, destructive interference 


\section{ABSTRACT}

UV resonance Raman scattering is uniquely sensitive to the molecular electronic structure as well as intermolecular interactions. To better understand the relationship between electronic structure and resonance Raman cross section, we carried out combined experimental and theoretical studies of neutral tyrosine and the tyrosinate anion. We studied the Raman cross sections of four vibrational modes as a function of excitation wavelength and we analyzed them in terms of the contributions of the individual electronic states as well as of the Albrecht A and B terms. Our model, which is based on time-dependent density functional theory (TDDFT), reproduced the experimental resonance Raman spectra and Raman excitation profiles for both studied molecules with good agreement. We found that for the studied modes the contributions of Albrecht's B terms in the Raman cross sections were important across the frequency range spanning the $\mathrm{L}_{\mathrm{a}, \mathrm{b}}$ and $\mathrm{B}_{\mathrm{a}, \mathrm{b}}$ electronic excitations in tyrosine and tyrosinate anion. Furthermore, we demonstrated that interference with high energy states had a significant impact and could not be neglected even when in resonance with a lower-energy state. Symmetry of the vibrational modes served as an indicator of the dominance of the A or B mechanisms. Excitation profiles calculated with a damping constant estimated from linewidths of the electronic absorption bands had the best consistency with experimental results. 


\section{INTRODUCTION}

UV Resonance Raman Scattering (RRS) spectroscopy is a powerful analytical technique permitting sensitive detection of hazardous materials as well as of structural change within biological systems. For example, RRS from aromatic amino acids such as tryptophan and tyrosine serves as an indicator of the local environment within a protein, ${ }^{1}$ such as ligand binding $^{2-3}$. UV-RRS was used to measure hydrophilic interactions, in particular the H-bond strength between water solvent molecules and the polar groups on the aromatic side chain. ${ }^{4-7}$ RRS has also been used to investigate the interactions between metal atoms in metallo-enzymes ${ }^{8-}$ ${ }^{10}$ and nearby amino acid residues serving as coordinating ligands. ${ }^{10-12}$ Moreover, RRS has been used for examining tertiary protein structure such as the $\alpha$ and $\psi$ angles of the peptide linkages in proteins, ${ }^{13}$ as well as the hydrophobicity around aromatic amino acid residues. ${ }^{14}$ Clearly, the RRS signal is sensitive to intermolecular interactions or any process that affects the electronic structure. Because of the wide applicability of RRS for the study of interactions in biological environments, a fundamental understanding of how the signal responses of vibrational modes within a molecule are impacted by the electronic structure is important. The impact can be reflected in both frequency shifts as well as subtle changes in Raman intensities. ${ }^{13,15-17}$

The impact of molecular electronic structure on the resonance Raman signature can be seen through the anti-resonance phenomenon, where the Raman intensity is much less than to be expected based on the optical absorption. Anti-resonances are thought to occur ${ }^{18-22}$ when preresonance of a strongly allowed electronic transition destructively interferes with the resonance with a weakly allowed electronic transition. Destructive interference in tryptophan results in a "window" around $205 \mathrm{~nm}$ with reduced Raman return that enables studies of RRS of amide bonds with minimal interference. ${ }^{20}$ Theoretical modeling is essential to understanding of anti- 
resonance phenomena in RRS spectra. While it is not experimentally possible to probe in detail the connection between the RRS and the electronic structure, e.g., detect electronic transitions deep in the vacuum UV or probe weakly allowed and unresolved transitions, it is possible to model the electronic structure in detail. The electronic structure of molecules can be simulated with experimentally-validated TDDFT models, and the influences of the individual electronic states on the RRS return can be studied in the model.

The sum-over-states approach ${ }^{23}$ is a necessary framework for understanding how the different electronic states affect the Raman cross sections of given normal modes, and it readily explains structure in the absolute Raman peak intensity as a function of excitation wavelength, that is, excitation profile. Excellent examples are demonstrated in the studies of several systems, including benzene, ${ }^{22,}{ }^{24-26}$ substitute benzenes, ${ }^{27}$ formamide, ${ }^{18} \mathrm{ClO}_{2},{ }^{28}$ and tryptophan. ${ }^{20} \mathrm{~A}$ number of studies has focused on the analyzing the excitation profile in terms of the Albrecht A term. For benzene, which is the primary chromophore of tyrosine, the Albrecht A term preresonance approximation was used to demonstrate that contributions from strongly allowed electronic states deep in the UV (ca. $119 \mathrm{~nm})^{19}$ dominated any contributions from the lowest energy allowed transition at $183 \mathrm{~nm}$. Hildebrandt et al., ${ }^{5}$ and Patapoff et al. ${ }^{28}$ evaluated the A term of the sum-over-states expression using vibrational wavefunctions to calculate the FranckCondon overlap integrals in their sum of states calculation, and demonstrated interference effects. An analysis including the B term mechanism would be useful since non-symmetric modes can have significant contributions to the RRS spectrum, however the calculation of the Albrecht B term is theoretically complicated. While the A term involves a single summation over the electronic states, the B term involves a double summation over both the electronic and vibronic states. A simplified approach is desirable. 
To simulate the resonant Raman spectra with a reduced computational burden while obtaining insight into the influence of the electronic structure on the Raman intensities, we utilized the simplified sum-over-states approach that some of us introduced in Rappoport et al. ${ }^{29}$, where we replace the double summation over vibronic and electronic states by a single summation over electronic states. It is based on an approximate closure over the vibrational states belonging to each individual electronic state. The only required properties of the excited electronic states are their excitation energies, transition dipole moments, and their respective gradients along the (ground-state) vibrational modes. The complicated calculation of the FranckCondon factors is avoided, and the calculation of the resonance Raman of amino acids in solution becomes much more tractable. It should also be noted that there are several other theoretical approaches currently available, such as the method of Gaff et al. based on the geometry differences between the excited and ground states ${ }^{30}$ as well as time dependent short time approximation approach of Heller and coworkers ${ }^{31-32}$ as implemented by Jensen et al. in the Amsterdam Density Functional Code (ADF) $)^{33-36}$ and NWCHEM $^{37}$. A similar approach is implemented in Orca. ${ }^{38}$ We utilize the simplified sum over states approach of Rappoport because it enables us to analyze the contributions of the Albrecht A and B terms to the excitation profile separately as well as examine the contributions from individual electronic transitions to the resonance Raman cross section. This is not possible in the time dependent short time approximation approach, which makes use of the closure of the sum over electronic states.

The focus of this study is the RRS of tyrosine for three reasons. First, tyrosine is biologically very important in enzyme function as stated above. Second, tyrosine possesses an aromatic side chain that enables it to experience resonance enhancement independently from most other amino acids, at readily accessible UV wavelengths. Third, this amino acid has been 
examined extensively with a number of experimental studies reported that can be used to validate modeling results. Therefore, a detailed theoretical analysis, including the B term, combined with experimental studies, helps to shed additional light on the influence of dark or weakly allowed electronic states. Using the experimental data from this work and previous studies, ${ }^{39-42}$ we establish the accuracy of the simplified sum-over-states approach ${ }^{29}$ and use it to analyze the contributions of the individual electronic states as well as Albrecht $\mathrm{A}$ and $\mathrm{B}$ terms ${ }^{23}$ to resonance Raman spectra and Raman excitation profiles.

\section{METHODS}

Experimental

The solution phase samples for the cross section measurements were prepared by dissolving the material of interest in ultrapure water. To mitigate the photodegradation of the tyrosine solutions, the samples were magnetically stirred in small glass cups during the measurements, so that the laser beam was continuously exposed to fresh sample. The cups were covered with UV-transparent quartz cover slips to prevent evaporation of the acetonitrile internal standard.

The UV resonance Raman spectroscopy measurements were carried out with an in-house built spectrometer system. The UV laser light was generated with two different laser systems. A Coherent Sabre FreD frequency-doubled argon-ion laser was used to generate $229 \mathrm{~nm}$ and 244 nm CW excitation light. A Crystalaser frequency-quadrupled pulsed Nd:YLF laser was used to generate $262 \mathrm{~nm}$ radiation with a high-repetition rate $(\sim 3 \mathrm{kHz})$ and low pulse energies. In all cases the incident laser power on the sample averaged $\sim 10-50 \mathrm{~mW}$. The excitation radiation was weakly focused on the sample with a $150 \mathrm{~mm}$ focal length lens. A $15 \times$ reflecting objective 
(Edmund Optics ReflX, N.A. $=0.5$, working distance $24.5 \mathrm{~mm}$ ) was used to collect the Raman scattered light. A backscattering configuration was used in which the laser beam was directed onto the sample by a right angle mirror attached to the central obscuration of the reflecting objective. An additional $150 \mathrm{~mm}$ lens focused the Raman scattered light on the spectrometer entrance slit. The spectrometer slit width was set to $100 \mu \mathrm{m}$. UV-grade quartz lenses and aluminum mirrors were used in all cases to steer and focus the laser beam and collect the Raman scattered light. The Rayleigh scattered excitation light was filtered by long-pass filters (Barr Associates) designed for each excitation wavelength. A Princeton Instruments SP2500 triple grating spectrometer $(0.5 \mathrm{~m}$ focal length, $\mathrm{f} / 6.2)$ was used to disperse the Raman scattered light. For the measurements described here, a 2400 grooves $/ \mathrm{mm}$ grating blazed at $300 \mathrm{~nm}$ was used. The light was detected using a PI/Acton Pixis 2K thermoelectrically-cooled UV-enhanced CCD camera $(2048 \times 512$ pixels). The Raman shift was calibrated for each excitation wavelength by measuring known bands of acetonitrile and cyclohexane. Blank spectra were collected at each excitation wavelength and subtracted from the RRS spectra.

UV-Vis absorption measurements were performed using a Thermo Evolution $60 \mathrm{UV}$-Vis spectrometer to obtain the absorbance in the wavelength range from 190-800 $\mathrm{nm}$. The samples were measured in a $1 \mathrm{~cm}$ path length quartz cell, with a concentration of $0.02 \mathrm{mg} / \mathrm{ml}$ for tyrosine and for the tyrosinate anion. The absorbance values obtained were then converted to molar absorptivities. In addition to general guidance for the resonance Raman spectroscopy measurements, the absorption measurements provide parameters necessary to correct for absorption effects on the Raman cross section values.

HPLC grade acetonitrile (Sigma-Aldrich) was used as the cross section standard, where the cross section values for acetonitrile were obtained from the work of Asher et al. ${ }^{43}$ The cross 
sections for acetonitrile can be modeled in the deep-UV using the modified Albrecht A term formula

$$
\sigma_{R}=K_{1} v_{0}\left(v_{0}-v_{j}\right)^{3}\left[\frac{v_{e}^{2}+v_{0}^{2}}{\left(v_{e}^{2}+v_{0}^{2}\right)^{2}}+K_{2}\right]^{2},
$$

where $\sigma_{R}$ is the Raman cross section, $v_{0}$ is the laser frequency in $\mathrm{cm}^{-1}, v_{j}$ is the mode vibrational frequency in $\mathrm{cm}^{-1}$, and $K_{1}, K_{2}$, and $v_{e}$ are empirically determined fitting parameters. ${ }^{43}$ For the $919 \mathrm{~cm}^{-1} \mathrm{C}-\mathrm{C}$ stretching band of acetonitrile $\left(\mathrm{CH}_{3} \mathrm{CN}\right)$, the parameter values are $K_{1}=2.26 \times 10^{-25}$ $\mathrm{cm}^{2} / \mathrm{sr}, k_{2}=0$, and $v_{e}=390000 \mathrm{~cm}^{-1}$. Using the internal standard technique, the cross section for the material of interest can be determined using the formula ${ }^{44}$

$$
\sigma_{R}^{S}=\sigma_{R}^{r}\left(\frac{I^{S}}{I^{r}}\right)\left(\frac{E\left(v_{0}-v_{j}^{r}\right)}{E\left(v_{0}-v_{j}^{S}\right)}\right)\left(\frac{C^{r}}{C^{S}}\right)\left(\frac{\varepsilon_{S}+\varepsilon_{e x c}}{\varepsilon_{r}+\varepsilon_{e x c}}\right),
$$

where $\sigma_{R}$ is the Raman cross section, $I$ is the integrated Raman band intensity, $E$ is a factor that takes into account the combined spectrometer and detector efficiencies as a function of wavelength, $C$ is the concentration, and $\varepsilon$ is the molar absorptivity of the resonant analyte of interest. Here the superscripts $r$ and $s$ refer to the acetonitrile reference and the sample of interest, respectively. The subscript exc refers to the excitation wavelength. The combined spectrometer and detector efficiencies were determined using a calibrated deuterium lamp (Ocean Optics DH-2000CAL). The last term in parentheses on the right-hand side of the equation is a factor that is used to correct for wavelength dependent UV self-absorption by the resonant analyte.

Theoretical 
Within the simplified sum-over-states approach, the resonance Raman cross section is represented as a sum over excited electronic states and is obtained by straightforward differentiation of the sum-over-states expansion of the frequency-dependent polarizability with respect to a vibrational normal mode. ${ }^{29}$ The differential Raman scattering cross section for a given normal mode $Q$ can be written as

$$
\left(\frac{\partial \sigma}{\partial \Omega}\right)_{Q}=\frac{\left(\omega-\omega_{Q}\right)^{4}}{2 \omega_{Q} c^{4}}\left|\left\langle\sigma_{Q}(\omega)\right\rangle\right|^{2},
$$

where $\omega$ is the excitation frequency, $\omega_{Q}$ is the vibrational frequency of mode $Q, c$ is the speed of light, and $\left\langle\boldsymbol{\sigma}_{Q}(\omega)\right\rangle$ is the angle-averaged Raman scattering tensor. The Cartesian component $m n$ of the Raman scattering tensor is computed as

$$
\begin{gathered}
\sigma_{Q}^{m n}(\omega)=\sum_{k}\left[-\mu_{0 k}^{m} \mu_{0 k}^{n}\left[\frac{\left(\Omega_{\mathrm{k}}-\omega\right)^{2}-\gamma_{k}^{2}}{\left(\left(\Omega_{\mathrm{k}}-\omega\right)^{2}+\gamma_{k}^{2}\right)^{2}}+\frac{2 i\left(\Omega_{\mathrm{k}}-\omega\right)^{2} \gamma_{k}}{\left(\left(\Omega_{\mathrm{k}}-\omega\right)^{2}+\gamma_{k}^{2}\right)^{2}}\right] \frac{\partial \Omega_{\mathrm{k}}}{\partial Q}+\left[\mu_{0 k}^{m} \frac{\partial \mu_{0 k}^{n}}{\partial Q}+\right.\right. \\
\left.\left.\frac{\partial \mu_{0 k}^{m}}{\partial Q} \mu_{0 k}^{n}\right]\left[\frac{\Omega_{\mathrm{k}}-\omega}{\left(\Omega_{\mathrm{k}}-\omega\right)^{2}+\gamma_{k}^{2}}+\frac{i \gamma_{k}}{\left(\Omega_{\mathrm{k}}-\omega\right)^{2}+\gamma_{k}^{2}}\right]\right]
\end{gathered}
$$

where $\Omega_{k}$ is the electronic state excitation energy of the $k^{\text {th }}$ electronic state, $\mu_{0 k}{ }^{m}$ and $\mu_{0 k}{ }^{n}$ are the $m$ and $n$ components of the $0 \rightarrow k$ transition dipole moment, and $\gamma_{k}$ is its linewidth. There are some key features in the above expression. First, the two terms in equation (4) are analogous to the Albrecht A and B terms. ${ }^{23}$ As with the Albrecht A term, the first term in equation (4) has the derivative of the excitation energy $\Omega_{k}$ with respect to the normal coordinate $Q$ which is only nonzero for symmetric vibrational modes. Second, as the excitation frequency $\omega$ approaches the excitation energy $\Omega_{k}$, there is resonance with one of the excited electronic states and that state is expected to dominate the Raman intensity, which potentially allows us to restrict the summation to the excited states near or below the excitation frequency $\omega$. The linewidth $\gamma_{k}$ of the $k^{\text {th }}$ excited 
electronic state cannot be computed from first principles. Thus, we approximated $\gamma_{k}$ in resonance Raman spectra by the linewidth fitted from the experimental absorption spectrum $(\gamma=0.2 \mathrm{eV})$. To demonstrate the effect of linewidth, spectra are calculated with $50 \mathrm{meV}, 100 \mathrm{meV}, 200 \mathrm{meV}$, and $300 \mathrm{meV}$ linewidth for neutral tyrosine. We optimized the ground-state structures using density functional theory with B3LYP hybrid functional ${ }^{45}$ and triple-zeta basis sets TZVPP. ${ }^{46}$ The structure optimizations included the conductor-like solvation model (COSMO) with the effective dielectric constant for water of $\varepsilon=80 .{ }^{47}$ Subsequently, vibrational modes, transition dipole derivatives and excitation energy gradients were computed by numerical differentiation of the ground-state energy gradients, transition dipoles, ${ }^{48-49}$ and excitation energies ${ }^{50}$ respectively, including the effect of solvation, while the frequencies of electronic transitions and transition dipoles were computed analytically without COSMO. All calculations were performed using the TURBOMOLE suite of programs, version 6.0..$^{51-52}$ In order to compare computed sum-over-states RRS with another approach, we calculated the RRS of neutral tyrosine using the time-dependent approach of Jensen et al. ${ }^{33}$ as implemented in the Amsterdam Density Functional (ADF) code. Following the approach of Jensen and Schatz we utilized the BP86 ${ }^{53-55}$ functional and the TZP $\mathrm{P}^{56}$ (triple $\zeta$ plus polarization functions Slater type orbitals basis set). Spectra were calculated with the AORESPONSE ${ }^{35-36}$ module using linewidths of 0.007 Hartrees as well as using the COSMO model to account for solvation in water.

\section{RESULTS AND DISCUSSION}

We used the simplified sum-over-states approach within the framework of TDDFT to investigate the influence of the molecular electronic structure on the RRS cross section. To this end, we first establish the accuracy of the simplified sum-over-states approach for tyrosine and 
tyrosinate by comparing the theoretical predictions to experimental UV-Vis absorption spectra, RRS spectra measured with $229 \mathrm{~nm}$ and $244 \mathrm{~nm}$ excitation, and excitation profiles from this work and Refs. 40-42.

\section{UV-Vis Spectra}

We compare the theoretical and experimental UV-Vis spectra of tyrosine and tyrosinate in Figure 1. For the neutral tyrosine, the form of the predicted curve generally follows the experimental measurement, where there are three broad features that have been assigned to the $\mathrm{B}_{\mathrm{a}, \mathrm{b}}, \mathrm{L}_{\mathrm{a}}$ and $\mathrm{L}_{\mathrm{b}}$ electronic transitions, with maxima at $193 \mathrm{~nm}, 223 \mathrm{~nm}$, and $275 \mathrm{~nm}$. Molar absorptivities of $\varepsilon_{275}=1370 \mathrm{~L} \mathrm{~mol}^{-1} \mathrm{~cm}^{-1}$ and $\varepsilon_{223}=8050 \mathrm{~L} \mathrm{~mol}^{-1} \mathrm{~cm}^{-1}$ were measured, which compare well with literature values ${ }^{57}$. From the full width at half-maximum (FWHM) of the features of the UV-Vis absorption spectrum, a damping constant of $0.2 \mathrm{eV}(200 \mathrm{meV})$ was determined and used in subsequent theoretical calculations. The largest difference between the theoretical and experimental results occurs in the predicted absorption of the $\mathrm{L}_{b}$ transition of $\sim 0.4$ $\mathrm{eV}$, and differences of this magnitude are typical for TDDFT calculations. For the tyrosinate at $\mathrm{pH} 13$ the agreement between theory and experiment is even better, where the differences are $\sim 0.1 \mathrm{eV}$ for the $\mathrm{L}_{\mathrm{a}}$ and $\mathrm{L}_{\mathrm{b}}$ transitions, and $\sim 0.3 \mathrm{eV}$ for the $\mathrm{B}_{\mathrm{a}, \mathrm{b}}$ transitions, at $293 \mathrm{~nm}, 240 \mathrm{~nm}$, and $198 \mathrm{~nm}$. The experimental UV-Vis absorption spectrum replicates the red shift in the predicted electronic absorption curve. Molar absorptivities of $\varepsilon_{239}=7390 \mathrm{~L} \mathrm{~mol}^{-1} \mathrm{~cm}^{-1}$ and $\varepsilon_{293}=2000 \mathrm{~L}$ $\mathrm{mol}^{-1} \mathrm{~cm}^{-1}$ were measured for the tyrosinate anion. 


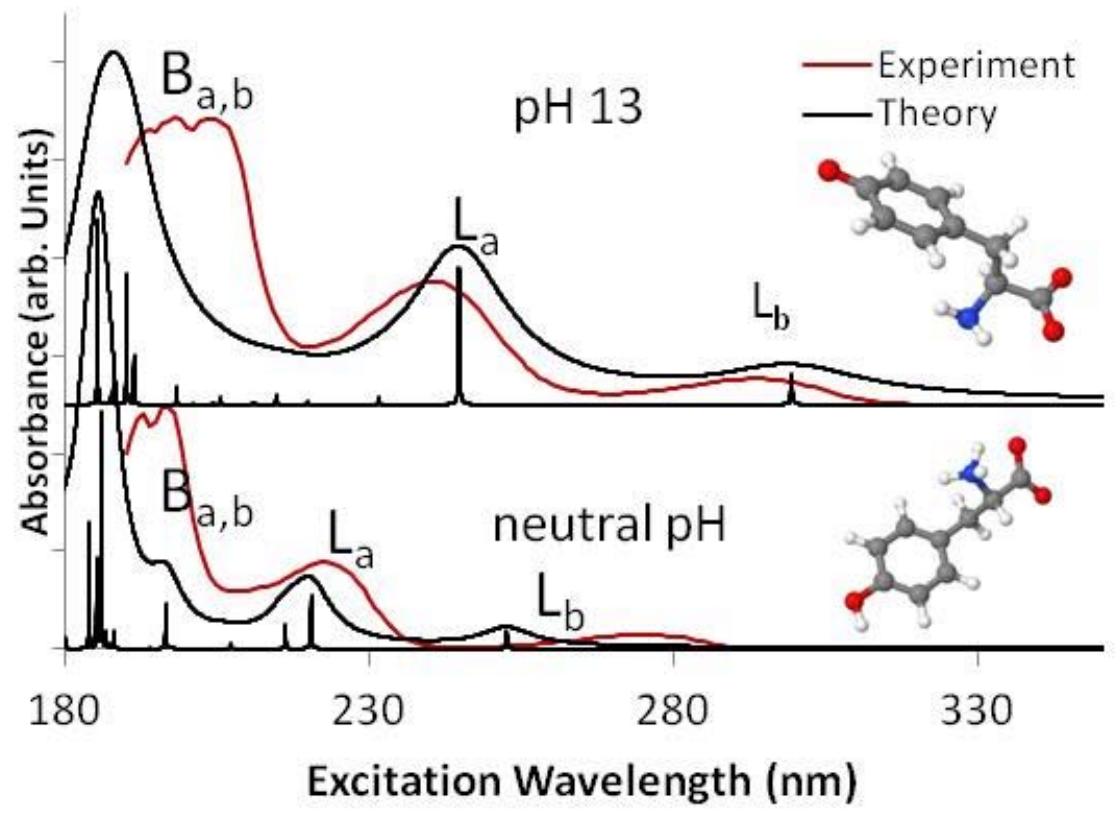

Figure 1: Comparison of experimental UV-Vis absorption spectra and TDDFT theoretical calculations for neutral tyrosine and tyrosinate at $\mathrm{pH} 13$, on the same scale. The general shapes of the theoretical curves follow the same form as the experimental data. Data from experiment is shown in red and data from TDDFT calculations are shown in black. As shown in the ball and stick models, the hydroxyl oxygen on the tyrosine ring and the carboxylate functional group are deprotonated at $\mathrm{pH} 13$ for a -2 anion. At neutral $\mathrm{pH}$ the amine group is protonated, the carboxylate functional group is deprotonated, and the hydroxyl group on the tyrosine ring is intact. The net molecular charge is neutral.

Resonance Raman spectra

The second validation point for the theoretical models of the two forms of tyrosine is the comparison of theoretical and experimental RRS spectra. Figure 2 shows the comparison of theoretical and experimental RRS spectra with $229 \mathrm{~nm}$ and $244 \mathrm{~nm}$ laser excitation. Excellent agreement in terms of relative intensities is obtained for neutral tyrosine with $229 \mathrm{~nm}$ excitation. Peaks are easily assigned to the $8 \mathrm{a}, 8 \mathrm{~b}$, ring-O stretch, ring-C stretch, $9 \mathrm{a}$, and a ring breathing mode. We attribute a shoulder at $1670 \mathrm{~cm}^{-1}$ in the experimental spectra to water molecule $\mathrm{H}-\mathrm{O}-$ $\mathrm{H}$ bending, sharing intensity with the $8 \mathrm{a}$ ring $\mathrm{C}-\mathrm{C}$ stretching mode. Except for the excited-state 
linewidth $\gamma$, which was fitted to the experimental absorption spectrum, no empirical or scaling factors were applied in the calculation of the RRS. Because of the harmonic approximation in the determination of vibrational frequencies, theoretical wavenumbers are typically $30-40 \mathrm{~cm}^{-1}$ greater than the experimental ones. Similarly, the theoretical spectrum for the $244 \mathrm{~nm}$ excitation matches the experimental spectrum very well. For tyrosinate with $244 \mathrm{~nm}$ excitation, similar agreement between theory and experiment is obtained. The removal of the phenoxy hydrogen produces a red-shift of the $8 \mathrm{a}$ and $8 \mathrm{~b}$ peaks, although the theoretical model overestimates the magnitude of the frequency shift for the $8 \mathrm{~b}$ mode. The peak at $1667 \mathrm{~cm}^{-1}$ is again attributed to intensity sharing with solvent water molecules. For tyrosinate with $229 \mathrm{~nm}$ there is good agreement, except for a single peak at $1376 \mathrm{~cm}^{-1}$, which corresponds to a ring-O stretch. Compared to neutral tyrosine, its frequency is predicted to be blue-shifted by about $100 \mathrm{~cm}^{-1}$, which is mostly due to the strengthening of the $\mathrm{C}-\mathrm{O}$ bond. We assume that this mode is not observed in experimental spectra due to its sensitivity to the local environment.

For comparison to the time-dependent finite lifetime approach, calculated RRS spectra from ADF with $229 \mathrm{~nm}$ and $244 \mathrm{~nm}$ excitation for tyrosine are presented in Figure 2. Spectra calculated with ADF are generally similar to the results of the sum-over-states calculation, except the relative intensities of the $9 \mathrm{a}$, ring- $\mathrm{C}$, and ring-O modes are different, and a ring twist mode around $1294 \mathrm{~cm}^{-1}$ becomes prominent. The differences in the vibrational frequencies are due to the use of the non-hybrid BP86 functional with ADF. Although the sum-over-states results are truncated after summation over 20 states, due to the similarity with the closed form finite lifetime calculation, we conclude this is a reasonable number of states. 
We also note that the theoretical Raman spectra contain a peak at the same position as the $825 \mathrm{~cm}^{-1}$ peak found in the experimental spectrum. This peak is assigned in the literature to an overtone of the 16a mode in Fermi resonance with the ring breathing mode at $854 \mathrm{~cm}^{-1}$.
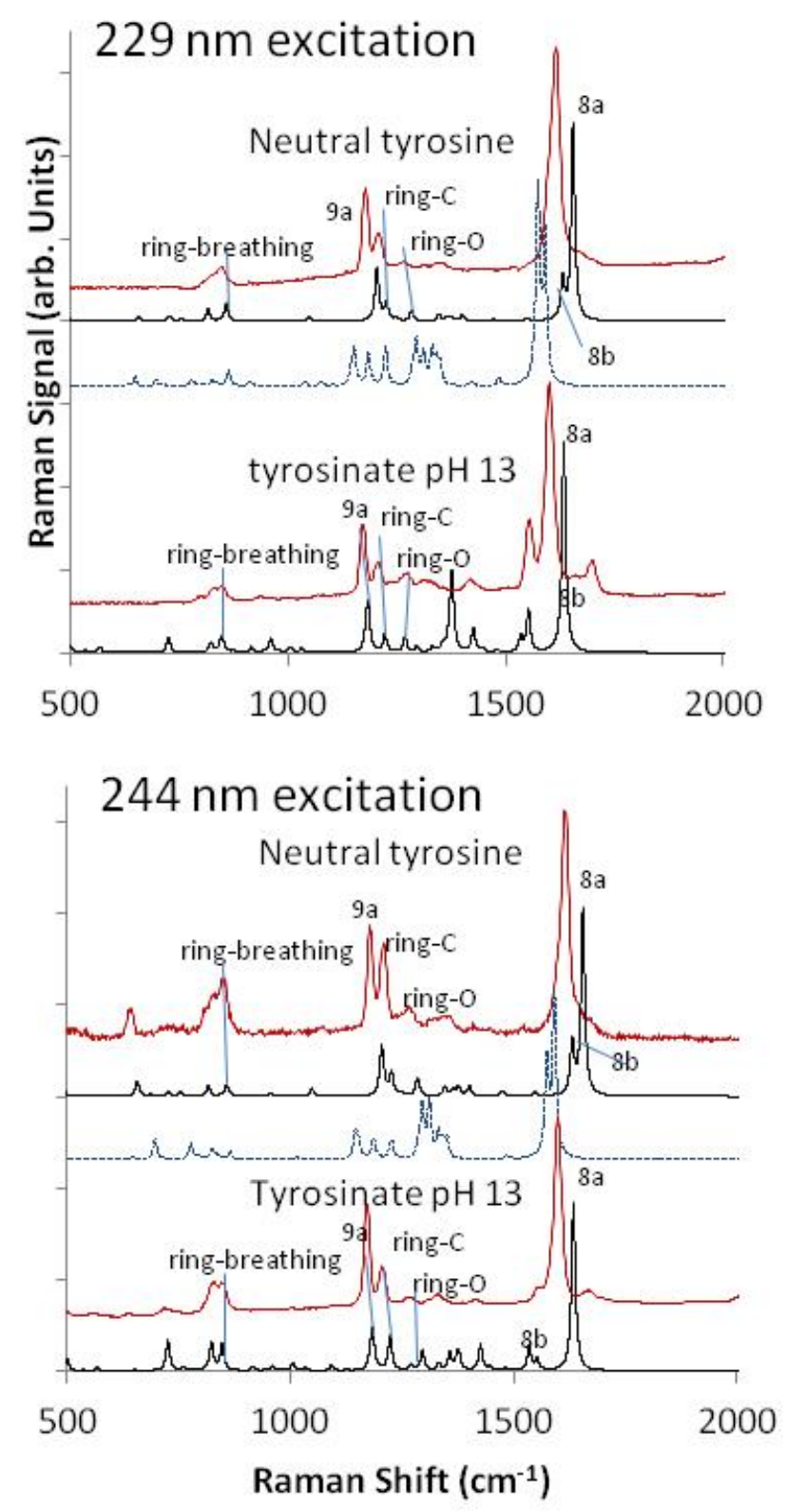

Figure 2: Comparison of theoretical and experimental RRS spectra with $229 \mathrm{~nm}$ and 244 $\mathrm{nm}$ laser excitation. Red traces represent experimental measurements, the black traces represent the results of the simplified sum-over-states calculations, and the blue dashed lines represent the results of the time-dependent approach as implemented in the Amsterdam Density Functional (ADF) code for comparison. 
Raman excitation profiles

We compare theoretical predictions for the absolute Raman cross sections to experimental measurements from this study and Refs. 40-42. Figure 3 shows the excitation profiles, that is, theoretical absolute Raman cross sections as a function of excitation wavelength, to the experimental measurements, for the $9 \mathrm{a}$, ring- $\mathrm{C}$ stretch mode, and the $8 \mathrm{a}$ modes. The literature values come from Fodor ${ }^{40}$, Ludwig ${ }^{41}$, and Asher ${ }^{42}$, and absolute cross sections are measured in this study at $229 \mathrm{~nm}, 244 \mathrm{~nm}$, and $262 \mathrm{~nm}$ for tyrosine and tyrosinate. For the $8 \mathrm{a}$ vibration at $1613 \mathrm{~cm}^{-1}$ with $229 \mathrm{~nm}$ (this study and Fodor) and $230 \mathrm{~nm}$ (Ludwig, 8a and 8b intensities reported together), there is good agreement $\left(5.1 \cdot 10^{-25}, 3.4 \cdot 10^{-25}\right.$, and $4.1 \cdot 10^{-25}$ $\mathrm{cm}^{2} / \mathrm{sr} \cdot$ molecule, respectively). Although other overlapping data points from Fodor and Ludwig agree well, the maximum value in the Ludwig data does not appear in the measured trend in the Fodor data. The same can be said for the $1180 \mathrm{~cm}^{-1}$ excitation profile. However, the data from Fodor does show a local minimum around a $218 \mathrm{~nm}$ excitation and a local maximum at $230 \mathrm{~nm}$. For the neutral tyrosine, the theoretical models follow the form of the experimental excitation profiles well, when the data points from all three studies are considered. For example, there is excellent agreement on the cross over point with respect to wavelength where the ring-C stretch mode at $1210 \mathrm{~cm}^{-1}$ comes to dominate the 9a mode at $1180 \mathrm{~cm}^{-1}(202 \mathrm{~nm}$ theoretical vs. $204 \mathrm{~nm}$ experiment). However, the theoretical excitation profiles consistently underestimate the experimental cross sections across the excitation wavelengths considered. For the neutral tyrosine and wavelengths shorter than $220 \mathrm{~nm}$, the theoretical absolute cross section is less than an order of magnitude less than the experimental value, and for wavelengths longer than $220 \mathrm{~nm}$, the theoretical absolute cross section underestimates the experiment by about a factor of five. 
The experimental data compare well with each other with good agreement for overlapping values of Asher and Ludwig. The $229 \mathrm{~nm}$ and the $244 \mathrm{~nm}$ data points from this study follows the experimental trend in the previous data very well, but the $262 \mathrm{~nm}$ data points for tyrosinate, especially for the $1208 \mathrm{~cm}^{-1}$ and $1601 \mathrm{~cm}^{-1}$ peaks vary significantly from the 260 $\mathrm{nm}$ excitation values from Asher. For the tyrosinate anion at $\mathrm{pH} 13$, the theoretical excitation profiles compare well with the experimental data. Again, the experimental absolute cross sections follow the shapes of the theoretical excitation profile curves. Based on the good agreement between the theoretical and experimental UV-Vis spectra, RRS spectra, and Raman excitation profiles for both neutral tyrosine and tyrosinate, we conclude that that the simplified sum-over-states approach provides a reasonable description for the Raman response in the two forms of tyrosine and can be used to study the interference effects in their resonance Raman spectra.

Destructive interference

The crucial advantage of the simplified sum over states approach is that it allows investigation of the interference between contributions from different electronic states to the RRS excitation profiles. Figure 4 shows the comparison of the theoretical electronic absorption curve to the RRS excitation profiles predicted from the summation of 5, 10, 15, and 20 lowestenergy electronic states for the $9 \mathrm{a}$, ring-C stretch, $8 \mathrm{~b}$, and $8 \mathrm{a}$ modes. The summation over the five lowest lying electronic states includes the two transitions at $253 \mathrm{~nm}$ and $221 \mathrm{~nm}$ that we assigned to the $\mathrm{L}_{\mathrm{a}}$ and $\mathrm{L}_{\mathrm{b}}$ transitions in the TDDFT electronic excitation spectrum. In addition, the TDDFT calculations predict much weaker transitions between $190 \mathrm{~nm}$ and $205 \mathrm{~nm}$, and the strongly absorbing $\mathrm{B}_{\mathrm{a}, \mathrm{b}}$ transitions are predicted to fall within the $170-190 \mathrm{~nm}$ range. The effects 
of different electronic states on the total predicted RRS cross section are clearly visible for the investigated modes. As with Figure 1, three absorption features are apparent in the electronic absorption spectrum even in log scale. For the 9 a mode $\left(1204 \mathrm{~cm}^{-1}\right.$ harmonic frequency), the excitation profile from summing the 5 lowest-lying electronic states generally follows the two low-energy features of the electronic absorption spectrum, although the profile is strongly dominated by the feature around $220 \mathrm{~nm}$. Both the electronic absorption spectrum and the excitation profile for 5 states have a maximum at $220 \mathrm{~nm}$.

As five next higher-lying electronic states are added to the sum over states, the excitation profile increases, so that the excitation profile generally matches the maximum absorption at $\sim 185 \mathrm{~nm}$, and the absorption feature at $220 \mathrm{~nm}$ is reflected as a shoulder in the excitation profile. However, when additional excited states deep in the UV are included in the sum for a total of 15 states in the summation, two interesting observations can be made. First, the excitation profile relative to that determined for the 10 -state summation, is reduced across the entire excitation energy range considered. For excitation wavelengths close to the $220 \mathrm{~nm}$ feature we attribute to the $\mathrm{L}_{\mathrm{a}}$ absorption, the absolute cross sections are reduced by nearly a factor of four. The reduction in cross section approaches two orders of magnitude for excitation wavelengths far from the $220 \mathrm{~nm}$ feature. This is a surprising result because high-energy electronic states that are far from resonance with the optical excitation can have such a strong influence, even when the optical excitation is in resonance with a lower energy transition. The contribution of high-energy electronic states should not be neglected, especially when the oscillator strengths are much greater than the lower energy transitions. The terms in the sum over states corresponding to higher energy transitions have a considerable effect on the calculated cross section. Without 
inclusion of at least 15 electronic states, the agreement between the experimental and theoretical excitation profiles is clearly unsatisfactory.

Second, for additional electronic states, convergence seems to be reached with respect to the number of electronic states included in the sum since the excitation profile for 20 states is nearly identical to that obtained for 15 electronic states. Similar observations can be made from the comparison of the electronic absorption curve and the excitation profiles for the ring-C stretch, the $8 \mathrm{~b}$, and the $8 \mathrm{a}$ modes. For the ring-C stretch, most suggestively, instead of a local maximum at the $220 \mathrm{~nm}$, a local minimum at $217 \mathrm{~nm}$ appears for summations of 10, 15, and 20 states. This produces a shift of the local maximum for the summations of 15 and 20 states to 223 $\mathrm{nm}$. Although the excitation profile for a summation of 5 states produces a local maximum at $252 \mathrm{~nm}$, which we attribute to resonance with the $\mathrm{L}_{b}$ electronic transition, for summations of 15 states, a local minimum is produced. Based on the dependence of the predicted excitation profile on the electronic states included in the sum over states, we conclude that the theoretical model is showing destructive interference between different transition dipole moments to different electronic states. While excitation can be in direct resonance with a given electronic state, interference from more strongly absorbing states deep in UV can have a strong impact on the RRS cross section. Although it is possible that other states deeper in the UV may still have an effect, the summation over the 20 lowest electronic states including the $\mathrm{L}_{\mathrm{a}}, \mathrm{L}_{\mathrm{b}}$, and $\mathrm{B}_{\mathrm{a}, \mathrm{b}}$ transitions captures the behavior of the RRS spectra and excitation profiles sufficiently well. It should also be pointed out that the models give insight on the influence of very high-energy transitions that cannot be measured experimentally due to photoinduced degradation of the analyte molecule. 

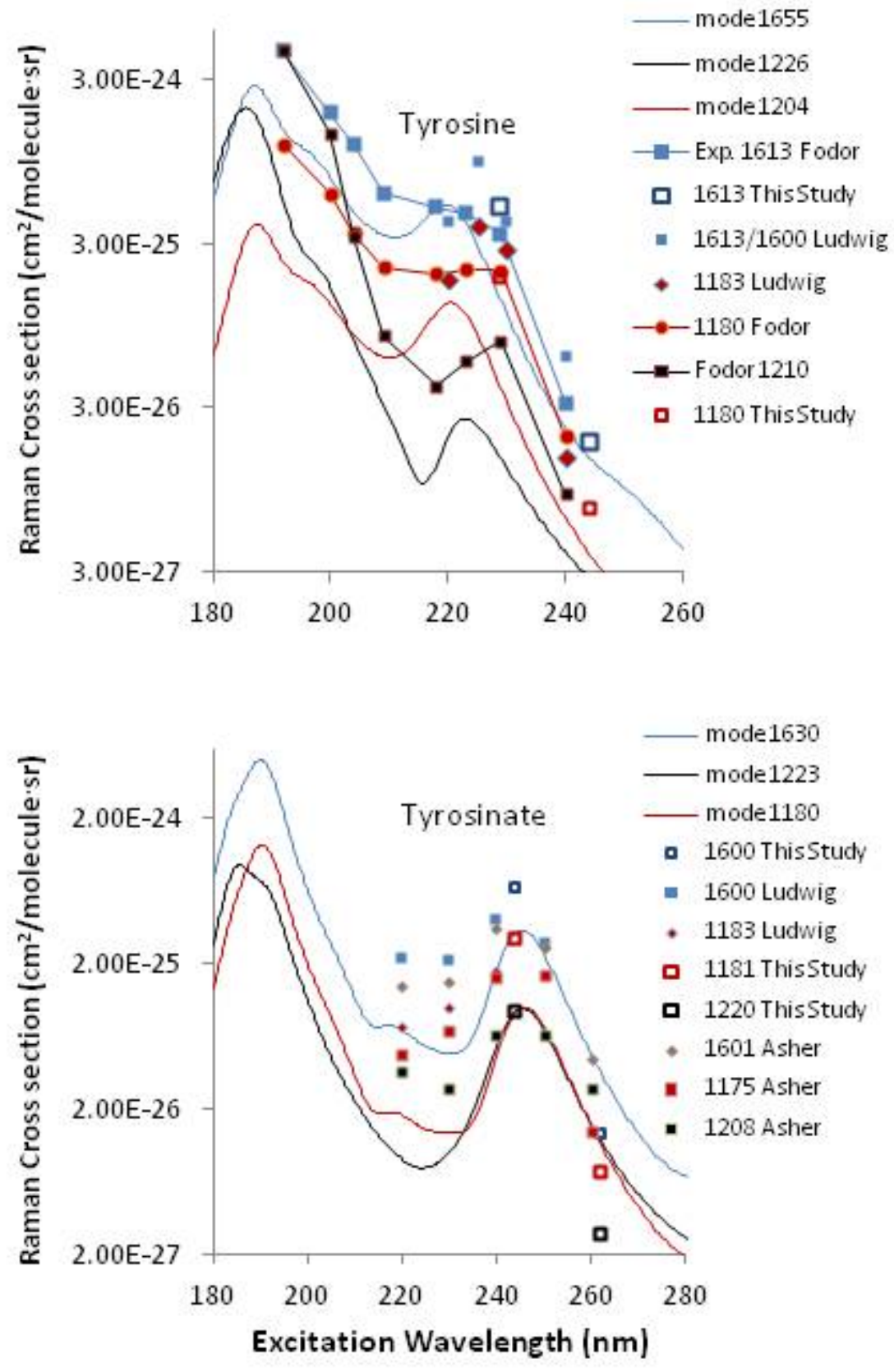

Figure 3: Comparison of experimental and theoretical excitation profiles (Raman cross section as a function excitation wavelength in the range of $180 \mathrm{~nm}$ to $280 \mathrm{~nm}$ ) of neutral tyrosine and tyrosinate anion. The solid curves represent the theoretical excitation profiles, labeled according to calculated vibrational frequency, and the symbols indicate experimental measurements. Lines joining experimental measurements are intended to aid the reader. The y-axis for both plots is in $\log$ scale. 
Figure 5 shows the comparison of the theoretical electronic absorption curve and the excitation profiles for the $9 \mathrm{a}$, ring- $\mathrm{C}$ stretch, $8 \mathrm{a}$, and $8 \mathrm{~b}$ modes of the tyrosinate anion in the range of $170 \mathrm{~nm}$ to $350 \mathrm{~nm}$. As with the tyrosine, the two lowest-energy transitions to the first two excited states dominate the excitation profiles for sums of 5 and 10 states for all four modes considered. Significant changes to the excitation profile, across the entire excitation wavelength range considered are demonstrated when higher-energy states are included in the sum for 15 and 20 states. For all four modes, maximum Raman cross section is obtained from the sum of 15 states, and the reduction in Raman cross section between the sum for 15 and 20 states demonstrates destructive interference. Unlike the results for tyrosine, self-consistency was not obtained for sums of 15 states. This is not surprising given the redshift of the theoretical electronic absorptions as well as increase in oscillator strength of the two lowest energy transitions $L_{a}$ and $L_{b}$ shown in the stick spectra in Figure 1. However, the same conclusion drawn for the neutral tyrosine can be made, namely that preresonance with states high in energy have a non-negligible contribution, even when in resonance with low energy states. 


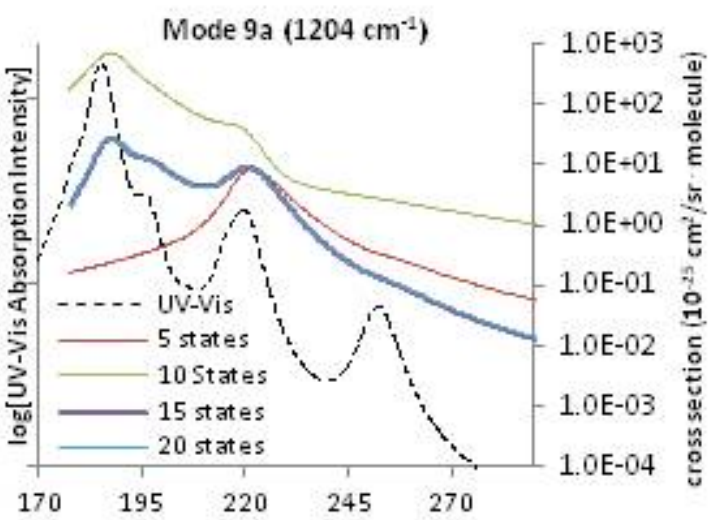

Excitation Wavelength $(\mathrm{nm})$

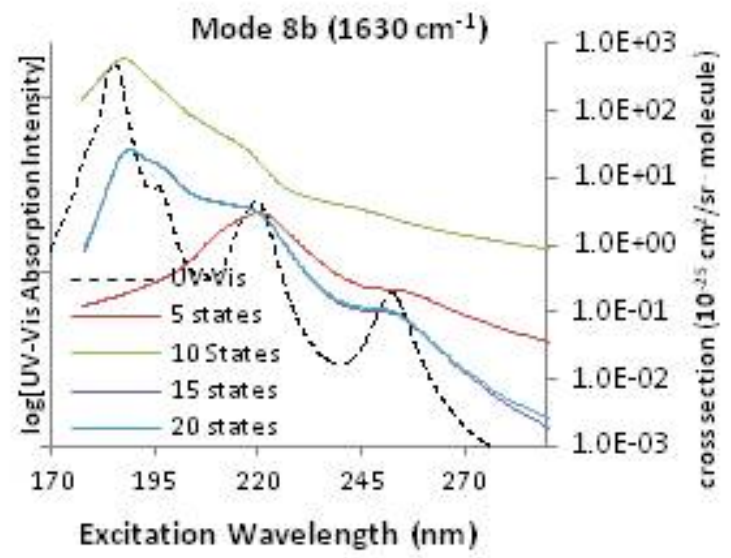

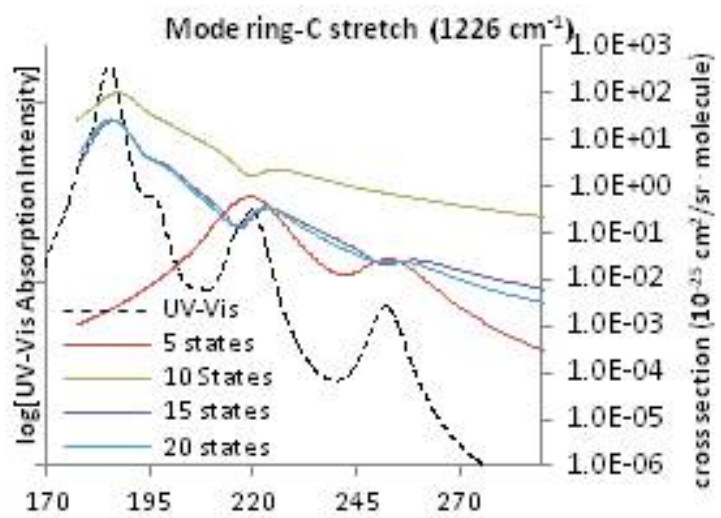

Excitation Wavelength $(\mathrm{nm})$

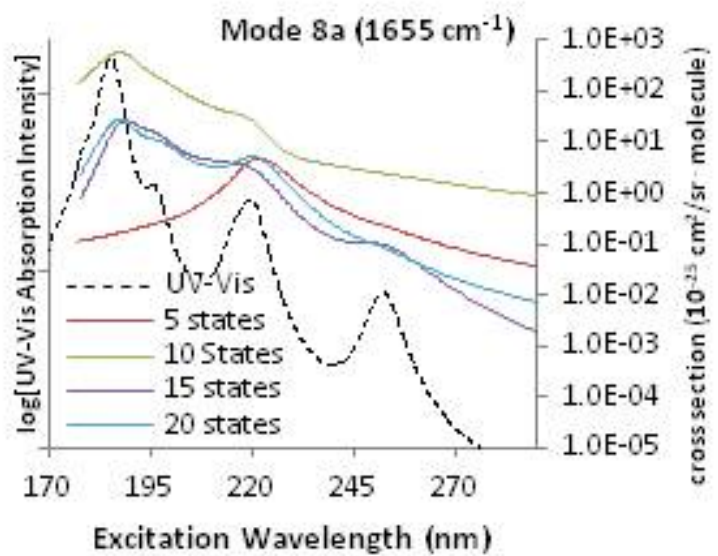

Excitation Wavelength $(\mathrm{nm})$

Figure 4: Comparison of the excitation profiles of the $9 \mathrm{a}$, ring-C stretch, $8 \mathrm{~b}$, and $8 \mathrm{a}$ modes of neutral tyrosine for summations of 5, 10, 15, and 20 electronic states to the theoretical electronic absorption curve. All y-axes are on log scale. 

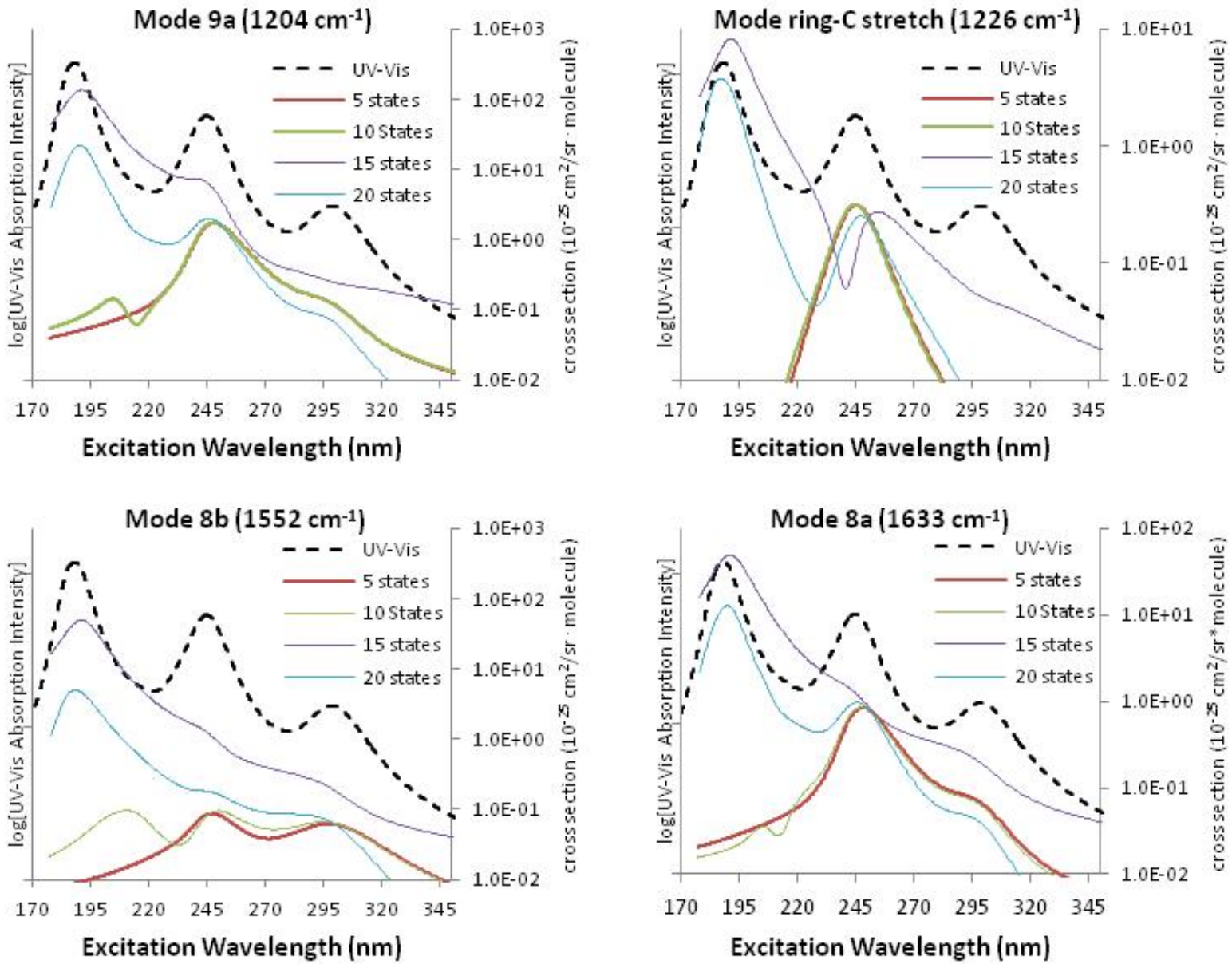

Figure 5: Comparison of the excitation profiles of the $9 \mathrm{a}$, ring-C stretch, $8 \mathrm{~b}$, and $8 \mathrm{a}$ modes of the tyrosinate anion for summations of 5, 10, 15, and 20 electronic states to the theoretical electronic absorption curve.

Contributions from the Albrecht A and B terms

The contributions from the A and B terms lend insight into the influence of the electronic structure on the RRS cross section and the Raman excitation profile. Figures 6 and 7 show the individual contributions of the A and B terms to the total cross sections for the $9 \mathrm{a}$, ring-C stretch, $8 \mathrm{~b}$, and $8 \mathrm{a}$ ring modes for neutral tyrosine and the tyrosinate anion. The y-axes are shown in $\log$ scale to display the broad range of cross sections. The excitation profiles are overlaid with the electronic absorption spectra to highlight the differences between the excitation profiles and the 
excitation spectrum. For the $9 \mathrm{a}, 8 \mathrm{~b}$, and $8 \mathrm{a}$ modes, the $\mathrm{B}$ term dominates RRS cross section for the excitation wavelengths considered. The symmetry of the vibrational modes can explain this observation, since it is known that symmetric vibrations dominate the contribution to the A term, and that $\mathrm{B}$ terms can receive contribution from non-symmetric modes. Figure 8 shows displacement vector representations of the neutral tyrosine, and it can be seen that the presence of the phenoxy hydrogen and oxygen greatly disrupts the symmetry of the vibrations. It should also be noted that for the ring- $\mathrm{C}$ stretch, $8 \mathrm{~b}$, and $8 \mathrm{a}$ vibrational modes, the $\mathrm{A}$ term contributions faithfully follow the electronic absorption curve for neutral tyrosine, except for the 9a mode which shows some destructive interference between the $\mathrm{L}_{b}$ and higher-energy electronic states. The B term contributions also generally follow the electronic excitation spectrum, except for the ring-C stretch mode, where strong destructive interference between the $\mathrm{L}_{\mathrm{a}}$ transition and other electronic states shows up as a local minimum.

The effect of vibrational symmetry on the A and B terms is more apparent in tyrosinate. As shown in Figure 9, removal of the phenoxy hydrogen at high $\mathrm{pH}$ increases the symmetry significantly, and this is reflected in the relative contributions to the excitation profiles from the $\mathrm{A}$ and $\mathrm{B}$ terms. For the $9 \mathrm{a}$, ring-C stretch, and the $8 \mathrm{a}$ modes, Figure 7 shows the $\mathrm{A}$ term is dominant when the excitation is close to resonance with the $\mathrm{L}_{\mathrm{a}}$ or $\mathrm{L}_{\mathrm{b}}$ electronic transition. This is not the case for the $8 \mathrm{~b}$ mode where the $\mathrm{B}$ mechanism remains the largest contributor to the excitation profile across all excitation wavelengths considered. An examination of the vibrational displacements for that mode shows the aromatic hydrogen bends on opposite sides of the phenoxylate oxygen point in opposite directions, making the mode non-symmetric. As a result, the A term contribution is limited for this particular mode. 
In Figure 7, there appears to be destructive interference between the $\mathrm{L}_{\mathrm{a}}$ transition and other excited electronic states in the B term contribution to the ring-C stretch mode. Although the TDDFT model has a transition at $220 \mathrm{~nm}$ and $216 \mathrm{~nm}$, there is a local minimum in the excitation profile at $218 \mathrm{~nm}$. However, the total RRS cross section is not greatly affected because the A term dominates at that wavelength, and the A term does not experience destructive interference between different terms in the sum over states. The excitation profile behavior for the $8 \mathrm{~b}$ vibrational mode also displays interesting behavior. While the A term is sensitive to the $\mathrm{B}_{\mathrm{a}, \mathrm{b}}$ and $\mathrm{L}_{\mathrm{a}}$ electronic transitions, it appears to get no contribution from the $\mathrm{L}_{\mathrm{b}}$ transition. For the $\mathrm{B}$ term, nearly the opposite is true where the excitation profile is barely affected by the $\mathrm{L}_{\mathrm{a}}$ transition, and clearly receives contributions from the $\mathrm{L}_{b}$ and $\mathrm{B}_{\mathrm{a}, \mathrm{b}}$ transitions. A similar observation can be made for the 8 a vibrational mode, where the A term strongly dominates the B term when in resonance with the transition to the $\mathrm{L}_{\mathrm{b}}$ electronic state, yet both the $\mathrm{A}$ and $\mathrm{B}$ terms experience enhancement when in resonance with the transition to the $\mathrm{L}_{\mathrm{a}}$ electronic state.

Considerations of the effects of broadening of the electronic transitions on the RRS spectrum

It is necessary to consider the effect of electronic transition broadening on the sum-overstates model in order to use the latter to understand interference effects on the RRS spectrum. In using the experimental linewidth in the calculation of the RRS spectrum for the damping constant in the sum over states approach, we assume that the linewidth arises only from homogeneous broadening mechanisms, and neglect inhomogeneous broadening, such as solvent effects, or other mechanisms. As shown in equation 4, the simplified sum over states utilized in this study only accounts for the effect of homogeneous broadening to the electronic absorptions. If mechanisms other than homogeneous broadening make a significant contribution to the 
experimental linewidth, then the true homogeneous broadening factor would be smaller than the $0.2 \mathrm{eV}$ used to calculate RRS spectra. In addition, inhomogeneous broadening also can change the lineshape of an electronic transition from a Lorentzian to a Gaussian or Voigt shape. Outside of the full width half max (FWHM), the Gaussian and Voigt lineshapes go more rapidly to zero than the Lorentzian lineshape. Thus, the effect of a significant contribution from an inhomogeneous broadening mechanism would be to reduce interference between electronic states in the sum-over-states. In addition to a reduction of interference between electronic transitions, a decrease in the homogeneous broadening damping factor would be to increase the predicted Raman cross section. It was noted above that the theoretical absolute Raman cross sections consistently underestimate the experimental values, and a smaller homogeneous damping factor may account for the consistent difference between theoretical prediction and experimental cross section values.

To demonstrate the effect of a smaller contribution of homogeneous broadening to the linewidth in the electronic spectrum on the sum over states model, and to provide a basis for comparison to experimental data, excitation profiles are calculated for a progression of damping constants. Figure 10 shows a comparison of calculated excitation profiles for the $8 \mathrm{a}, 8 \mathrm{~b}, 9 \mathrm{a}$, and ring-C vibrational modes for the values $0.05 \mathrm{eV}$ and $0.2 \mathrm{eV}$ of homogeneous broadening. For the $0.2 \mathrm{eV}$ and $0.3 \mathrm{eV}$ linewidths, the excitation profiles are nearly identical, and the excitation profile values for the $0.1 \mathrm{eV}$ linewidth fall midway between those for the $0.05 \mathrm{eV}$ and $0.2 \mathrm{eV}$ profiles. As expected, the result of the reduction of the linewidth is to reduce interference between contributions from individual electronic states to the Raman cross section.

The reduced interference shows itself in two ways. First, when the linewidth is reduced to $0.05 \mathrm{eV}$, the Raman cross section is enhanced by up to two orders of magnitude when in 
resonance with an electronic transition. Second, the excitation profile starts to follow the absorption spectrum more closely in that the $\mathrm{L}_{b}$ transition at $253 \mathrm{~nm}$ becomes significantly more apparent in the excitation profile. However, this will have a significant impact on the relative intensities between the ring-C, $8 \mathrm{a}$, and $9 \mathrm{a}$ peaks that is not consistent with experimental data. For the narrow $0.05 \mathrm{eV}$ linewidth, the ring- $\mathrm{C}$ mode receives significant enhancement in the vicinity of the $\mathrm{L}_{b}$ electronic transition so that on resonance it would be expected to dominate the 8 a peak by an order of magnitude and have equal intensity at $\sim 245 \mathrm{~nm}$. For the same electronic linewidth our model also predicts the ring- $\mathrm{C}$ mode should dominate the 9 a mode by nearly two orders of magnitude on resonance and should have equal intensity at $240 \mathrm{~nm}$. However, experimental excitation profile data for neutral tyrosine shown in Figure 3 shows that both the 8 a and $9 \mathrm{a}$ intensities consistently dominates the signal from the ring- $\mathrm{C}$ mode. The neutral tyrosine spectrum in Figure 2 from $244 \mathrm{~nm}$ excitation also shows the 9a still has slightly greater intensity than the ring- $\mathrm{C}$ mode. The relative peak intensities for the $8 \mathrm{a}, 9 \mathrm{a}$, and ring- $\mathrm{C}$ modes predicted using a damping factor of $0.2 \mathrm{eV}$ are more consistent with the experimental data than results obtained with damping factors of $0.05 \mathrm{eV}$ or $0.1 \mathrm{eV}$. We conclude that the assumption of homogeneous broadening as the dominant contributor to the electronic transition linewidth and the $\gamma=0.2 \mathrm{eV}$ linewidth are reasonable.

With respect to the consistent underestimation of the absolute RRS cross sections, it may be possible to adjust the damping factor to make the theoretical cross sections consistent with experimental ones. However, this is not justifiable without additional experimental data that illuminates the contribution from other electronic transition broadening mechanisms. 

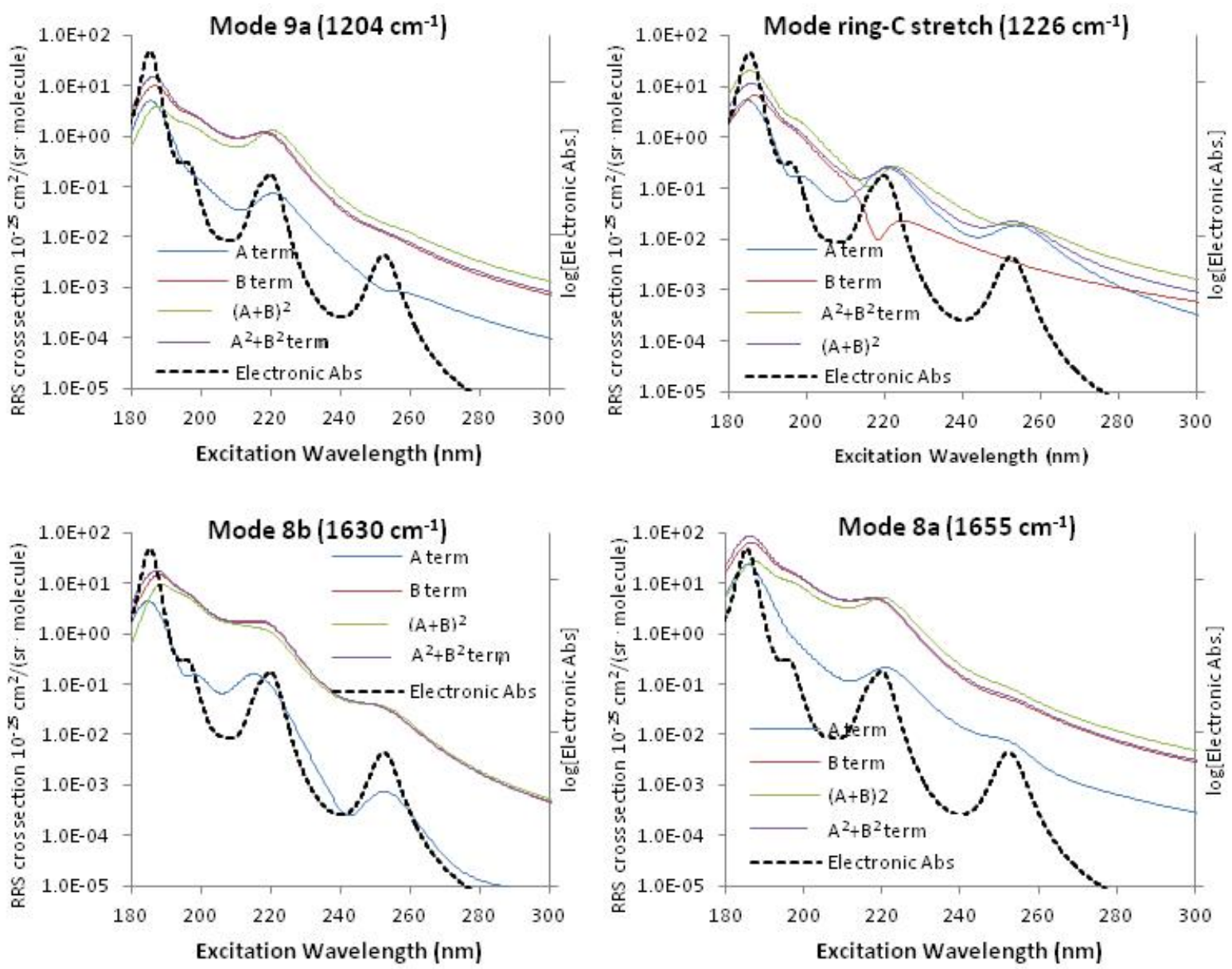

Figure 6: A comparison of the contributions from the A and B terms to the theoretical excitation profiles for the $9 \mathrm{a}$, ring-C stretch, $8 \mathrm{~b}$, and $8 \mathrm{a}$ modes of the neutral, zwitterionic form of tyrosine. The profiles that result from directly summing the A and $\mathrm{B}$ term polarizability tensor derivatives $(\mathrm{A}+\mathrm{B})^{2}$ and from summing the magnitudes while disregarding interference between $A$ and $B$ terms $\left(\mathrm{A}^{2}+\mathrm{B}^{2}\right)$ are plotted as well for each mode. 

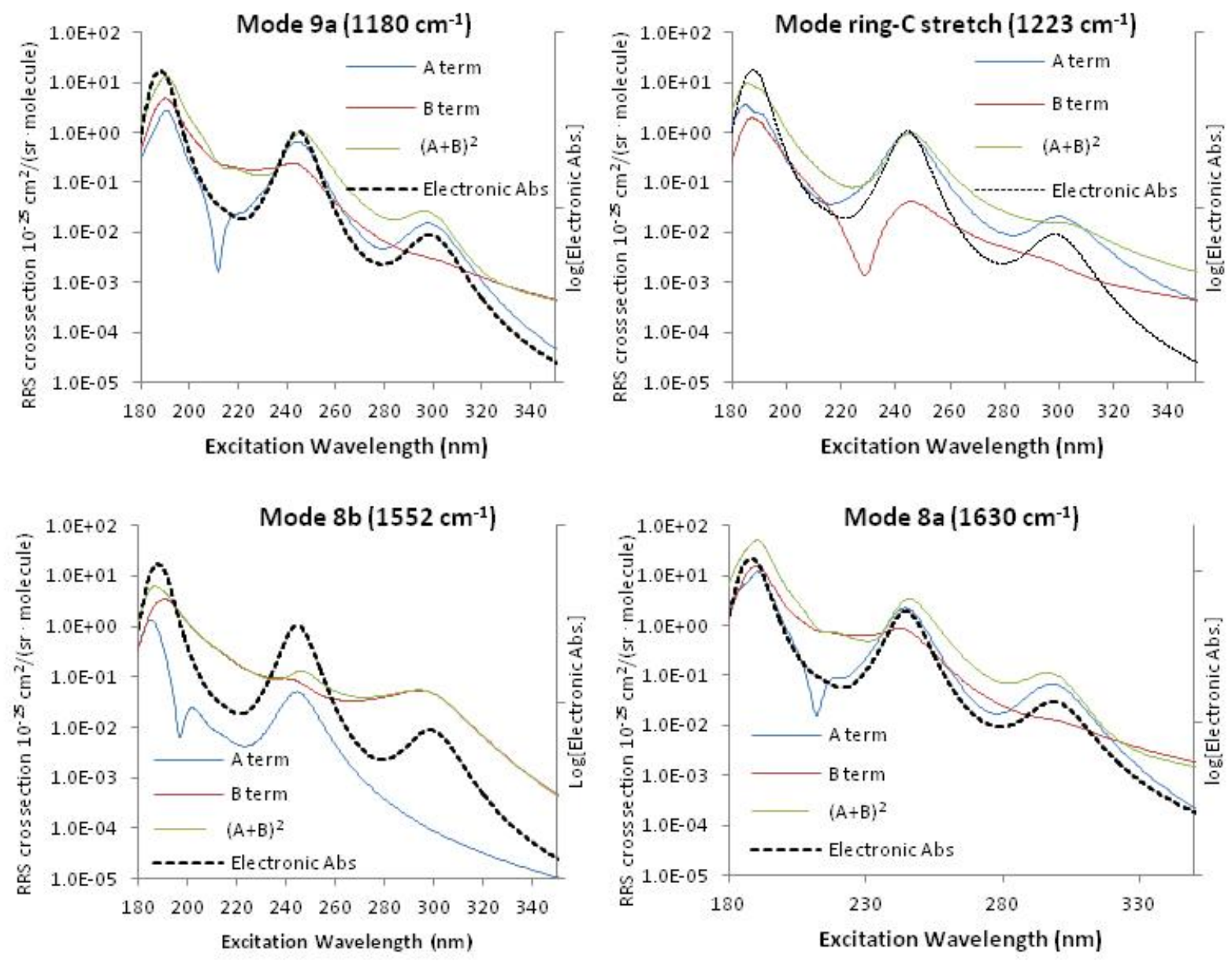

Figure 7: A comparison of the contributions from the A and B terms to the theoretical excitation profiles for the $9 \mathrm{a}$, ring-C stretch, $8 \mathrm{~b}$, and $8 \mathrm{a}$ modes of the of Tyrosinate anion. The profiles that result from directly summing the A and B term polarizability tensor derivatives $(\mathrm{A}+\mathrm{B})^{2}$ for each mode. 


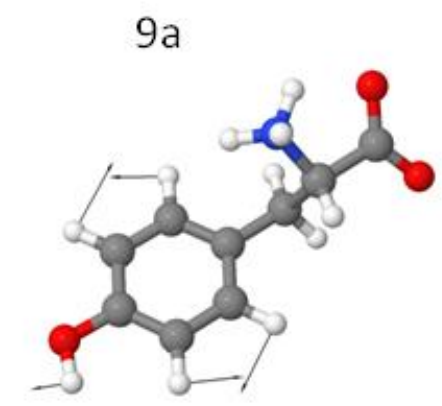

$8 b$

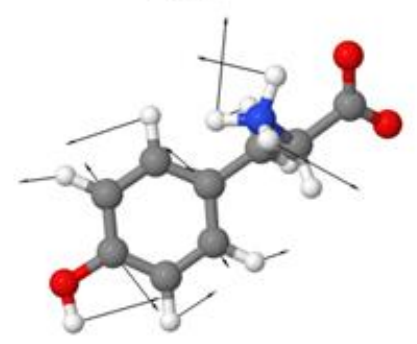

ring-C stretch

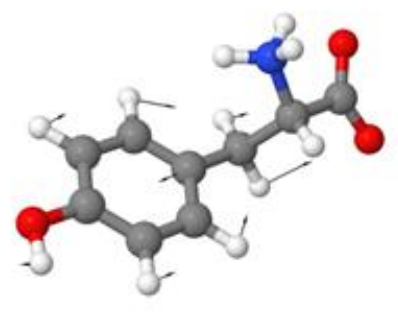

$8 \mathrm{a}$

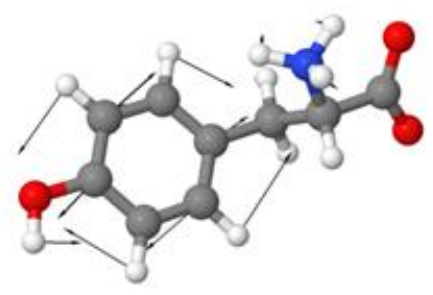

Figure 8: Vector representations of the $9 \mathrm{a}$, ring-C stretch, $8 \mathrm{~b}$, and $8 \mathrm{a}$ modes of zwitterionic, neutral Tyrosine. Note that the phenoxy hydrogen breaks the symmetry of the ring motions, so that for the $8 \mathrm{a}$ mode, the $\mathrm{C}-\mathrm{C}$ ring stretches are skewed relative to the ring oxygen-carbon axis.

\section{CONCLUSIONS}

The simplified sum-over-states approach has been used to study the influence of highenergy electronic states on the RRS spectrum of neutral tyrosine and tyrosinate anion at high $\mathrm{pH}$ with state-specific detail. Excellent agreement between theoretical calculations and experiment is obtained for not only the UV-Visible electronic absorption spectrum, but also for the RRS spectra and excitation profiles for the four vibrational modes considered with the electronic state linewidth, which is fitted to the experimental UV-Vis absorption spectrum. We establish the accuracy of the TDDFT model of tyrosine. From that model we are able to infer influence of high energy electronic states on the RRS response from tyrosine. First, although the optical excitation can be in resonance with lower energy electronic states, for some molecules it may not be advisable to neglect the contributions from high-energy states. For neutral tyrosine and the 
tyrosinate anion, even though transitions to the $\mathrm{B}_{\mathrm{a}, \mathrm{b}}$ electronic states are nearly $2 \mathrm{eV}$ greater in energy than the transition to the $\mathrm{L}_{b}$ electronic state, the destructive interference from these transitions can suppress the RRS response by orders of magnitude. Second, an examination of the contributions from the A and B Albrecht terms to the RRS cross sections shows that the symmetries of the vibrational modes affect the RRS excitation profile. Lastly, we examine the influence of homogeneous broadening of the electronic transitions on the RRS excitation profiles. We demonstrate that the excitation profiles calculated with a damping constant of 0.2 $\mathrm{eV}$ are more consistent with the experimental data and that our assumption of dominant homogeneous broadening is reasonable.

$9 a$

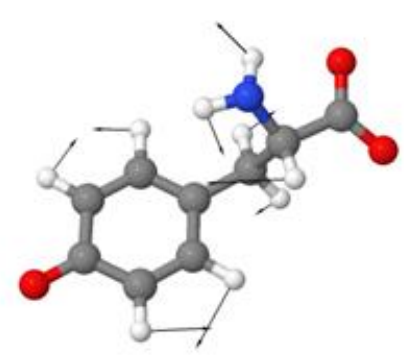

$8 b$

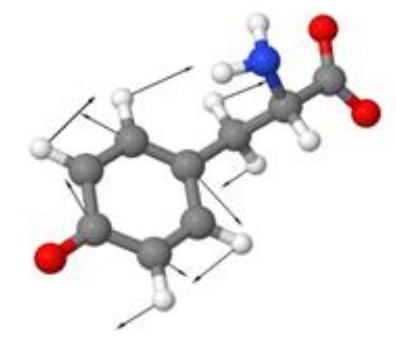

ring- $C$ stretch

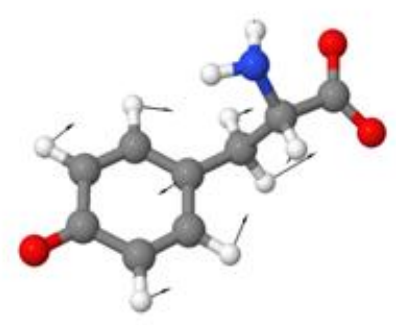

$8 a$

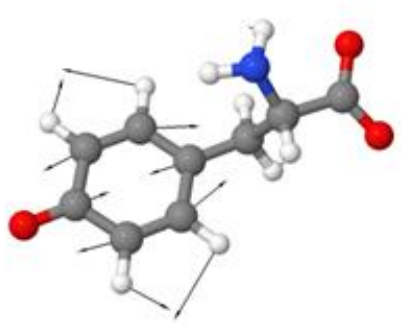

Figure 9: Vector representations of the 9a, ring-C stretch, $8 \mathrm{~b}$, and $8 \mathrm{a}$ modes of the tyrosinate anion. Note that the symmetry of the ring motions is increased by the removal of the phenoxy hydrogen, where the ring $\mathrm{C}-\mathrm{C}$ stretch for the $8 \mathrm{a}$ mode is now parallel to the ring oxygen-carbon axis. 

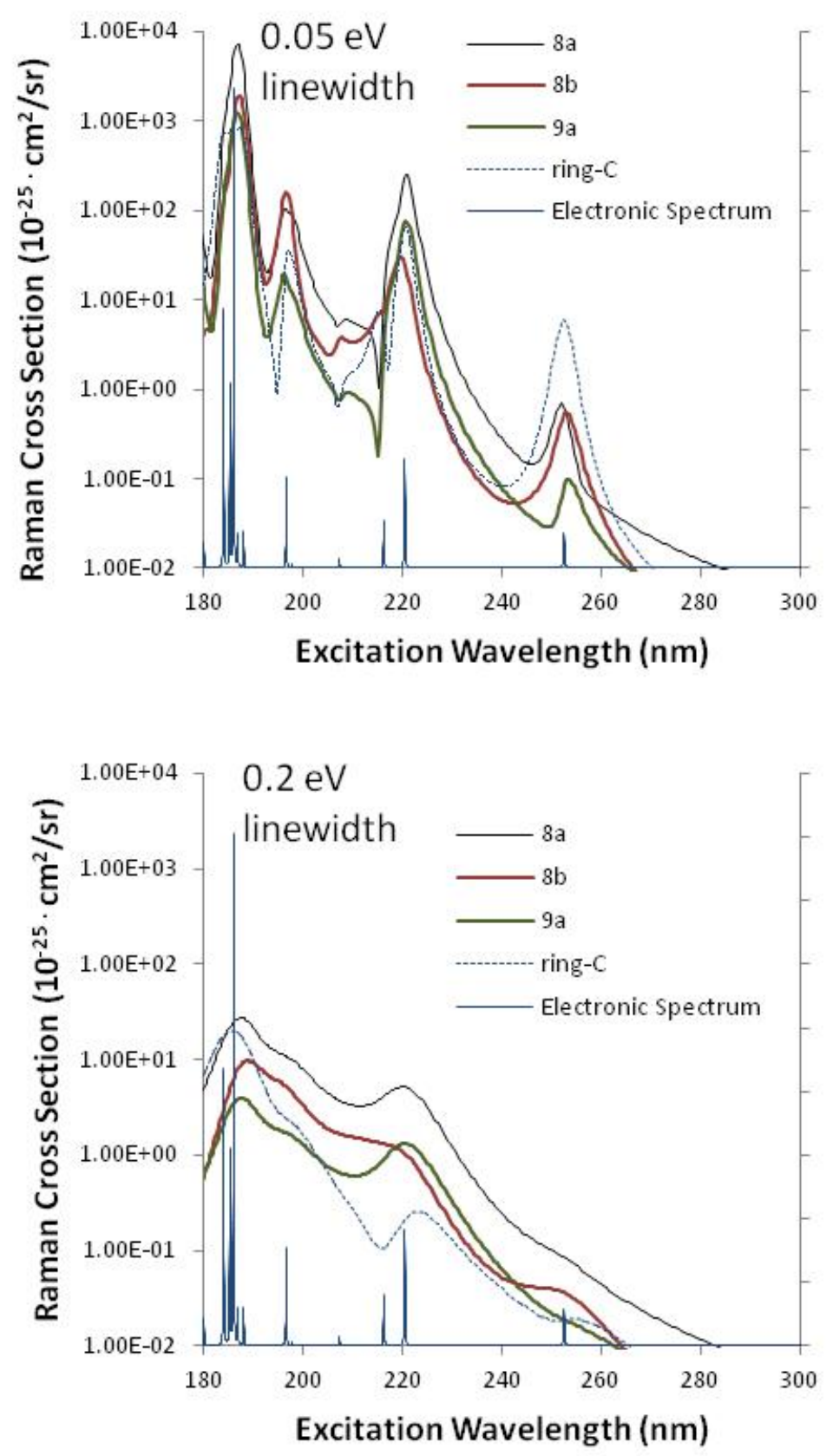

Figure 10: A comparison of the sum-over-states calculated excitation profiles for 0.05 $\mathrm{eV}$ and $0.2 \mathrm{eV}$ linewidths for the $8 \mathrm{a}, 8 \mathrm{~b}, 9 \mathrm{a}$, and ring- $\mathrm{C}$ modes. The electronic spectrum calculated with TDDFT is included for comparison. The values for the excitation profiles for $0.1 \mathrm{eV}$ linewidth are midway between the values for the $0.05 \mathrm{eV}$ and $0.2 \mathrm{eV}$ excitation profiles shown. The excitation profile values for the $0.3 \mathrm{eV}$ linewidth are nearly identical to those calculated for $0.2 \mathrm{eV}$. 


\section{ACKNOWLEDGEMENTS}

J.B.C. and E.D.E gratefully acknowledge funding provided by the Edgewood Chemical Biological Center's In-house Laboratory Independent Research (ILIR) program administered by Dr. A. W. Fountain. S.K.S. and A.A.-G. acknowledge the Defense Threat Reduction Agency grant HDTRA1-10-1-0046. D.R. and A.A.-G. acknowledge support through the Cyberdiscovery Initiative Type II (CDI2) grant of the National Science Foundation (NSF), grant number OIA1125087. A.A.-G. acknowledges generous support from the Corning Foundation. 


\section{REFERENCES}

(1) Kochendoerfer, G. G.; Kaminaka, S.; Mathies, R. A., Ultraviolet resonance Raman examination of the light-induced protein structural changes in rhodopsin activation, Biochem. 1997, 36 (43), 13153-13159.

(2) Huang, S. C.; Huang, J.; Kloek, A. P.; Goldberg, D. E.; Friedman, J. M., Hydrogen bonding of tyrosine b10 to heme-bound oxygen in Ascaris hemoglobin - Direct evidence from UV resonance Raman spectroscopy, Journal of Biological Chemistry 1996, 271 (2), 958-962.

(3) Nagai, M.; Imai, K.; Kaminaka, S.; Mizutani, Y.; Kitagawa, T., Ultraviolet resonance Raman studies of hemoglobin quaternary structure using a tyrosine-alpha 42 mutant: Changes in the alpha(1)beta(2) subunit interface upon the T->R transition, Journal of Molecular Structure 1996, $379,65-75$.

(4) Couling, V. W.; Foster, N. W.; Klenerman, D., Ultraviolet resonance Raman spectroscopic study of the average environment of tyrosine in native and denatured barnase, Journal of Raman Spectroscopy 1997, 28 (1), 33-38.

(5) Hildebrandt, P. G.; Copeland, R. A.; Spiro, T. G.; Otlewski, J.; Laskowski, M., Jr.; Prendergast, F. G., Tyrosine hydrogen-bonding and environmental effects in proteins probed by ultraviolet resonance Raman spectroscopy, Biochem. 1988, 27, 5426-33.

(6) Huang, S.; Huang, J.; Kloek, A. P.; Goldberg, D. E.; Friedman, J. M., Hydrogen bonding of tyrosine B10 to heme-bound oxygen in Ascaris hemoglobin. Direct evidence from UV resonance Raman spectroscopy, J Biol Chem 1996, 271, 958-62.

(7) Offenbacher, A. R.; Chen, J.; Barry, B. A., Perturbations of Aromatic Amino Acids Are Associated with Iron Cluster Assembly in Ribonucleotide Reductase, Journal of the American Chemical Society 2011, 133 (18), 6978-6988.

(8) Nagai, M.; Yoneyama, Y.; Kitagawa, T., Characteristics in tyrosine coordinations of four hemoglobins M probed by resonance Raman spectroscopy, Biochem. 1989, 28, 2418-22.

(9) Asher, S. A.; Larkin, P. J.; Teraoka, J., Ultraviolet resonance Raman and absorption difference spectroscopy of myoglobins: titration behavior of individual tyrosine residues, Biochem. 1991, 30, 5944-54.

(10) Yeh, S. R.; Couture, M.; Ouellet, Y.; Guertin, M.; Rousseau, D. L., A cooperative oxygen finding hemoglobin from Mycobacterium tuberculosis - Stabilization of heme ligands by a distal tyrosine residue, Journal of Biological Chemistry 2000, 275 (3), 1679-1684.

(11) Das, T. K.; Couture, M.; Lee, H. C.; Peisach, J.; Rousseau, D. L.; Wittenberg, B. A.; Wittenberg, J. B.; Guertin, M., Identification of the Ligands to the Ferric Heme of Chlamydomonas Chloroplast

Hemoglobin: Evidence for Ligation of Tyrosine-63 (B10) to the Heme, Biochem. 1999, 38, 15360-15368.

(12) Nagai, M.; Nagatomo, S.; Nagai, Y.; Ohkubo, K.; Imai, K.; Kitagawa, T., Near-UV Circular Dichroism and UV Resonance Raman Spectra of Individual Tryptophan Residues in Human Hemoglobin and Their Changes upon the Quaternary Structure Transition, Biochem. 2012, 51 (30), 5932-5941.

(13) Ma, L.; Ahmed, Z.; Mikhonin, A. V.; Asher, S. A., UV Resonance Raman Measurements of Poly-L-Lysines's Conformational Energy Landscapes: Dependence on Perchlorate Concentration and Temperature, Journal of Physical Chemistry B 2007, 111 (26), 7675-7680. 
(14) Clarkson, J.; Sudworth, C.; Masca, S. I.; Batchelder, D. N.; Smith, D. A., Ultraviolet resonance Raman study of the avidin biotin complex, Journal of Raman Spectroscopy 2000, 31 (5), 373-375.

(15) Nagatomo, S.; Nagai, M.; Kitagawa, T., A New Way To Understand Quaternary Structure Changes of Hemoglobin upon Ligand Binding On the Basis of UV-Resonance Raman Evaluation of Intersubunit Interactions, Journal of the American Chemical Society 2011, 133 (26), 10101-10110.

(16) Pieridou, G. K.; Hayes, S. C., UV resonance Raman spectroscopy of TTR(105-115): determination of the pKa of tyrosine, Phys. Chem. Chem. Phys. 2009, 11, 5302-5309.

(17) Huang, S. C.; Peterson, E. S.; Ho, C.; Friedman, J. M., Quaternary structure sensitive tyrosine interactions in hemoglobin: A UV resonance Raman study of the double mutant $\mathrm{rHb}$ (beta 99Asp->Asn, alpha 42Tyr->Asp), Biochem. 1997, 36 (20), 6197-6206.

(18) Hildebrandt, P.; Tsuboi, M.; Spiro, T. G., Ultraviolet resonance Raman spectroscopy of formamide: evidence for $\mathrm{n}-\pi^{*}$ interferences and intermolecular vibronic coupling, J. Phys. Chem. 1990, 94, 2274-9.

(19) Asher, S. A.; Johnson, C. R., UV resonance Raman excitation profile through the 1B2u state of benzene, J. Phys. Chem. 1985, 89, 1375-9.

(20) Sweeney, J. A.; Asher, S. A., Tryptophan UV resonance Raman excitation profiles, J. Phys. Chem. 1990, 94, 4784-91.

(21) Avila Ferrer, F. J.; Barone, V.; Cappelli, C.; Santoro, F., Duschinsky, Herzberg-Teller, and Multiple Electronic Resonance Interferential Effects in Resonance Raman Spectra and Excitation Profiles. The Case of Pyrene, Journal of Chemical Theory and Computation 2013, 9 (8), 35973611.

(22) Ziegler, L.; Albrecht, A. C., Raman scattering of benzene in the ultraviolet, J. Chem. Phys. 1977, 67, 2753-7.

(23) Albrecht, A. C., On the Theory of Raman Intensities, Journal of Chemical Physics 1961, 34 (1), 1476-1484.

(24) Korenowski, G. M.; Ziegler, L. D.; Albrecht, A. C., Calculations of resonance Raman cross sections in forbidden electronic transitions: scattering of the $992 \mathrm{~cm}-1$ mode in the $1 \mathrm{~B} 2 \mathrm{u}$ band of benzene, J. Chem. Phys. 1978, 68, 1248-52.

(25) Ziegler, L. D.; Albrecht, A. C., Preresonance Raman scattering of overtones: the scattering of two overtones of benzene in the ultraviolet, J. Raman Spectrosc. 1979, 8, 73-80.

(26) Ziegler, L. D.; Hudson, B., Resonance Raman scattering of benzene and benzene-d6 with $212.8 \mathrm{~nm}$ excitation, Journal of Chemical Physics 1981, 74, 982-992.

(27) Ziegler, L. D.; Hudson, B. S., Vibronic coupling activity in the resonance Raman spectra of alkyl benzenes, J. Chem. Phys. 1983, 79, 1134-7.

(28) Patapoff, T. W.; Turpin, P. Y.; Peticolas, W. L., The use of a Kramers-Kronig transform techniques to calculate the resonance Raman excitation profiles of anharmonic molecular vibrations, J. Phys. Chem. 1986, 90, 2347-51.

(29) Rappoport, D.; Shim, S.; Aspuru-Guzik, A., Simplified Sum-Over-States Approach for Predicting Resonance Raman Spectra. Application to Nucleic Acid Bases, Journal of Physical Chemistry Letters 2011, 2 (11), 1254-1260.

(30) Gaff, J. F.; Franzen, S.; Delley, B., Ab Initio Calculation of Resonance Raman Cross Sections Based on Excited State Geometry Optimization, Journal of Physical Chemistry A 2010, 114 (43), 11681-11690. 
(31) Heller, E. J.; Sundberg, R.; Tannor, D., Simple aspects of Raman scattering, J. Phys. Chem. 1982, 86, 1822-33.

(32) Lee, S.-Y.; Heller, E. J., Time-dependent theory of Raman scattering, J. Chem. Phys. 1979, $71,4777-88$.

(33) Jensen, L.; Zhao, L. L.; Autschbach, J.; Schatz, G. C., Theory and method for calculating resonance Raman scattering from resonance polarizability derivatives, Journal of Chemical Physics 2005, 123 (17).

(34) Zhao, L. L.; Jensen, L.; Schatz, G. C., Pyridine-Ag-20 cluster: A model system for studying surface-enhanced Raman scattering, Journal of the American Chemical Society 2006, 128 (9), 2911-2919.

(35) Van Gisbergen, S. J. A.; Snijders, J. G.; Baerends, E. J., Application of time-dependent density functional response theory to Raman scattering, Chemical Physics Letters 1996, 259, 599.

(36) van Gisbergen, S. J. A.; Snijders, J. G.; Baerends, E. J., Implementation of time-dependent density functional response, Computer Physics Communications 1999, 118 (2-3), 119-138.

(37) Aquino, F. W.; Schatz, G. C., Time-Dependent Density Functional Methods for Raman Spectra in Open-Shell Systems, J. Phys. Chem. A 2014, 118, 517-525.

(38) Petrenko, T.; Neese, F., Analysis and prediction of absorption band shapes, fluorescence band shapes, resonance Raman intensities, and excitation profiles using the time-dependent theory of electronic spectroscopy, Journal of Chemical Physics 2007, 127, 164319/1-164319/15. (39) Rava, R. P.; Spiro, T. G., Resonance Enhancement in the Ultraviolet Raman Spectra of Aromatic Amino Acids, Journal of Physical Chemistry 1985, 89, 1856-1861.

(40) Fodor, S. P. A.; Copeland, R. A.; Grygon, C. A.; Spiro, T. G., Deep-Ultraviolet Raman Excitation Profiles and Vibronic Scattering Mechanisms of Phenylalanine, Tyrosine, and Tryptophan, Journal of the Americal Chemical Society 1989, 111 (15), 5509-5518.

(41) Ludwig, M.; Asher, S. A., Ultraviolet Resonance Raman Excitation Profiles of Tyrosine: Dependence of Raman Cross Sections on Excited-State Intermediates, Journal of the Americal Chemical Society 1988, 110 (4), 1005.

(42) Asher, S. A.; Ludwig, M.; Johnson, C. R., UV Resonance Raman excitation profiles of the aromatic amino acids, J. Am. Chem. Soc. 1986, 108, 3186-97.

(43) Dudik, J. M.; Johnson, C. R.; Asher, S. A., Wavelength Dependence of the Preresonance Raman Cross-Sections of $\mathrm{CH}_{3} \mathrm{CN}, \mathrm{SO}_{4}{ }^{2-}, \mathrm{ClO}_{4}^{-}$, and $\mathrm{NO}_{3}^{-}$, J. Chem. Phys. 1985, 82 (4), $1732-$ 1740 .

(44) Emmons, E. D.; Tripathi, A.; Guicheteau, J. A.; Fountain, A. W.; Christesen, S. D., Ultraviolet Resonance Raman Spectroscopy of Explosives in Solution and the Solid State, The Journal of Physical Chemistry A 2013, 117 (20), 4158-4166.

(45) Becke, A. D., Density-functional thermochemistry. III. The role of exact exchange, JCP 1993, $98,5648-5652$.

(46) Weigend, F.; Ahlrichs, R., Balanced basis sets of split valence, triple zeta valence and quadruple zeta valence quality for $\mathrm{H}$ to $\mathrm{Rn}$ : Design and assessment of accuracy, Phys. Chem. Chem. Phys. 2005, 7, 3297-3305.

(47) Klamt, A.; Schueuermann, G., COSMO: a new approach to dielectric screening in solvents with explicit expressions for the screening energy and its gradient, J. Chem. Soc., Perkin Trans. 2 1993, , 799-805.

(48) Furche, F.; Rappoport, D., Density functional methods for excited states: Equilibrium structure and electronic spectra, Theor. Comput. Chem. 2005, 16, 93-128. 
(49) Bauernschmitt, R.; Ahlrichs, R., Treatment of electronic excitations within the adiabatic approximation of time dependent density functional theory, Chem. Phys. Lett. 1996, 256, 454464.

(50) Furche, F.; Ahlrichs, R., Adiabatic time-dependent density functional methods for excited state properties, J. Chem. Phys. 2002, 117, 7433-7447.

(51) TURBOMOLE V6.5 2013, a development of the University of Karlsruhe and Forschungszentrum Karlsruhe GmbH, 1989-2007; available from http://www.turbomole.com. (52) Furche, F.; Ahlrichs, R.; Haettig, C.; Klopper, W.; Sierka, M.; Weigend, F., Turbomole, Wiley Interdiscip. Rev.: Comput. Mol. Sci. 2014, 4, 91-100.

(53) Perdew, J. P., Erratum: Density-functional approximation for the correlation energy of the inhomogeneous electron gas, Physical review B 1986, 34, 7406.

(54) Perdew, J. P., Density-functional approximation for the correlation energy of the inhomogeneous electron gas, Physical Review B 1986, 33, 8822(R).

(55) Becke, A. D., Density-functional exchange-energy approximation with correct asymptotic behavior, Phys. Rev. A: Gen. Phys. 1988, 38, 3098-100.

(56) Van Lenthe, E.; Baerends, E. J., Optimized Slater-type basis sets for the elements 1-118, J. Comput. Chem. 2003, 24, 1142-1156.

(57) Pace, C. N.; Vajdos, F.; Fee, L.; Grimsley, G.; Gray, T., How to measure and predict the molar absorption coefficient of a protein, Protein Science 1995, 4, 2411-2423. 